\title{
Current trends in operative treatment of carpometacarpal osteoarthritis: a survey of European hand surgeons
}

\author{
Qi Yin ${ }^{1} \cdot$ Merel J.-L. Berkhout ${ }^{2,3} \cdot$ Marco J. P. F. Ritt ${ }^{1,3}$
}

Received: 10 January 2019 / Accepted: 18 March 2019 / Published online: 10 April 2019

(C) The Author(s) 2019, corrected publication 2019

\begin{abstract}
Background Surgical practice patterns in operative treatment of carpometacarpal (CMC-1) osteoarthritis (OA) have been evaluated in the USA repeatedly. This study evaluates the current surgical patterns in the treatment of isolated CMC-1 OA among hand surgeons in Europe.

Methods An online survey was distributed to hand surgeons of participating member states of the Federation of European Societies for Surgery of the Hand (FESSH). Information regarding country of practice, years of experience and surgical treatment of choice was collected. Statistical analyses of correlations between demographics and treatment preferences were done using chi-squared testing.

Results A total of 444 replies were recorded. Trapeziectomy with ligament reconstruction and tendon interposition (LRTI) was opted by $46 \%$ of the respondents as a procedure of first choice, followed by prosthetic joint replacement (25\%) and trapeziectomy with interpositional arthroplasty (23\%). Among surgeons practicing in the Netherlands, $35 \%$ mentioned trapeziectomy without any complementary procedures as a surgical treatment of first choice. Prosthetic joint replacement is chosen by respectively $96 \%$ and $72 \%$ of the respondents in Belgium and France as a preferred surgical procedure.

Conclusions This European-wide study provides insight in the prevailing surgical management for isolated CMC-1 OA. Trapeziectomy with LRTI is the most popular procedure among the participating hand surgeons in Europe. Geographical trends seem to play an important role in the choice of preferred technique. Factors influencing the decision-making among surgeons practicing in different countries should be explored more closely in future studies.

Level of evidence Level IV, prognostic study.
\end{abstract}

Keywords Carpometacarpal $\cdot$ CMC osteoarthritis $\cdot$ Trapeziometacarpal $\cdot$ Survey

\section{Introduction}

Carpometacarpal (CMC-1) osteoarthritis (OA) may develop following primary or posttraumatic damage of articular cartilage or degeneration of the stabilizing ligament complex. As the disease progresses, this may lead to basal thumb pain,

Qi Yin

Q.Yin@vumc.nl

1 Department of Plastic, Reconstructive and Hand Surgery, Amsterdam UMC, Location VUmc, P.O. Box 7057, 1007 MB Amsterdam, Netherlands

2 Department of Plastic, Reconstructive and Hand Surgery, Alrijne Hospital, P.O. Box 4220, 2350 CC Leiderdorp, Netherlands

3 The Hand Clinic, Keienbergweg 8, 1101

GB Amsterdam, Netherlands instability and loss of function. In a study among a general population, minimal radiographic OA was determined in participants from the age group 30-44 years onwards. For participants older than 75 , mild to severe OA was present in $38 \%$ of the female participants and $27 \%$ of the male participants [1]. When conservative treatment fails, surgical treatment may be indicated. Procedures include trapeziectomy with or without interposition and with or without additional ligament suspension or reconstruction, CMC-1 arthrodesis, implant arthroplasty and a plethora of other procedures using autologous or artificial materials $[2,3]$.

Current surgical practice patterns in operative treatment of carpometacarpal (CMC-1) osteoarthritis (OA) have been evaluated in the USA repeatedly [4-6]. Yet, the surgical practice among European hand surgeons remains unknown. The main goal of this study is to explore the prevailing surgical procedures for isolated CMC-1 OA among hand surgeons in Europe. 


\section{Methods}

Permission was requested from all individual member states of the FESSH to survey the members of the national member society using an online survey sent by electronic mail. An online survey on scaphotrapeziotrapezoid OA and CMC-1 $\mathrm{OA}$ was constructed and included one multiple-answer multiple-choice question on the surgical management of CMC-1 OA. The option "other" with a write-in box was provided. When "prosthetic joint replacement" or "trapeziectomy with interpositional arthroplasty" was chosen as preferred surgical procedure, the respondent was asked to specify the used material in an additional question. Demographic questions consisted of two single-answer multiple-choice questions on the country of practice and years of experience as hand surgeon. The survey was concluded by a free-response item. The questions were compiled into an online survey using SurveyGizmo. A pilot group consisting of four experienced hand surgeons at the authors' institution was asked to evaluate question clarity and identify potential problems. An online link to the survey was sent by the national delegates of the participating countries of the FESSH to their members by mail. One reminder was asked to be distributed. Data was collected between 11 October 2017 and 11 April 2018. When appropriate, open-ended answers obtained from the write-in box were categorized into original responses. Frequency distributions were created for all response variables and bivariate cross-tabulations were used to compare treatment variables with demographic variables. Comparison between the subgroups was done using the Pearson chi-squared test. Statistical significance was set at $P$ less than 0.05 .

\section{Results}

The survey was electronically delivered to 21 participating countries in Europe. A total of 444 responses was recorded, from which 5 surgeons $(1.1 \%)$ mentioned not to treat CMC-1 OA surgically. The demographic distribution of the 439 surgeons that treat CMC-1 OA surgically is displayed in Tables 1 and 2. Most participating surgeons practice in Germany $(23 \%)$, the Netherlands (16\%), Switzerland (10\%), and France $(8.9 \%)$. A summary of the respondents' preferred surgical procedures for CMC-1 OA is listed in Table 3. As respondents could choose more than one procedure in this question, the sum of recorded responses is more than the total number of participants. Trapeziectomy with ligament reconstruction and tendon interposition (LRTI) is the most popular surgical technique, chosen by almost half of the respondents $(46 \%)$ as a procedure of first choice for CMC-1 OA. Compared to other participating countries, this procedure is more often opted by surgeons practicing in Turkey (74\% vs $44 \% ; P=0.005)$. Surgeons practicing in the Netherlands more
Table 1 Survey demographics: country of practice

\begin{tabular}{lrr}
\hline Country of practice & Response, $N=439$ & \% Total responses \\
\hline Austria & 25 & 5.7 \\
Belgium & 26 & 5.9 \\
Bulgaria & 3 & 0.7 \\
Czech Republic & 12 & 2.7 \\
Estonia & 6 & 1.4 \\
Finland & 4 & 0.9 \\
France & 39 & 8.9 \\
Germany & 99 & 22.6 \\
Greece & 22 & 5.0 \\
Hungary & 17 & 3.9 \\
Ireland & 1 & 0.2 \\
Italy & 2 & 0.5 \\
Latvia & 6 & 1.4 \\
Netherlands & 72 & 16.4 \\
Norway & 10 & 2.3 \\
Poland & 8 & 1.8 \\
Portugal & 3 & 0.7 \\
Romania & 1 & 0.2 \\
Sweden & 15 & 3.4 \\
Switzerland & 45 & 10.3 \\
Turkey & 23 & 5.2 \\
\hline
\end{tabular}

often mentioned to perform only trapeziectomy without any additional procedure compared to other countries (35\% vs $17 \% ; P=0.001)$. Approximately one-fourth of all surgeons perform trapeziectomy with interpositional arthroplasty (23\%), with the vast majority of the participants using tendon as interpositional material (89\%). Trapeziectomy with interpositional arthroplasty is especially popular in Switzerland compared to other countries (36\% vs $21 \% ; P=$ 0.031). Prosthetic joint replacement for CMC-1 OA is also performed by one-fourth of all participating surgeons (26\%), most of them using a metal on metal or a metal on polyethylene joint implant (76\%). Prosthetic joint replacement is chosen by respectively $96 \%$ and $72 \%$ of the respondents in Belgium and France as a procedure of first choice, which is more than in all other countries $(P<0.0001)$.

Table 2 Survey demographics: years of experience as hand surgeon

\begin{tabular}{lcc}
\hline $\begin{array}{l}\text { Years of experience } \\
\text { as hand surgeon }\end{array}$ & Response, $N=439$ & \% Total responses \\
\hline$\leq 5$ & 74 & 16.9 \\
$6-15$ & 179 & 40.8 \\
$16-25$ & 122 & 27.8 \\
$>25$ & 64 & 14.6 \\
\hline
\end{tabular}


Table 3 Preferred surgical procedures for CMC-1 OA, arranged by popularity

\begin{tabular}{|c|c|c|}
\hline Preferred surgical procedure & Response & $\begin{array}{l}\% \text { Responses of total } \\
\text { participants, } N=439\end{array}$ \\
\hline Trapeziectomy with LRTI & 202 & 46.0 \\
\hline Prosthetic joint replacement & 113 & 25.7 \\
\hline Metal on polyethylene/metal & 86 & 76.1 \\
\hline Pyrocarbon & 21 & 18.6 \\
\hline Bioresorbable & 3 & 2.7 \\
\hline Other & 3 & 2.7 \\
\hline Trapeziectomy with interpositional arthroplasty & 100 & 22.8 \\
\hline Tendon & 89 & 89.0 \\
\hline Capsule & 7 & 7.0 \\
\hline Other & 4 & 4.0 \\
\hline Trapeziectomy only & 93 & 21.2 \\
\hline Trapeziectomy with ligament reconstruction only & 82 & 18.7 \\
\hline Arthrodesis of MC I and trapezium & 33 & 7.5 \\
\hline Surgical denervation & 24 & 5.5 \\
\hline $\begin{array}{l}\text { Trapeziectomy with hematoma distraction only (temporary K-wire } \\
\text { fixation) }\end{array}$ & 20 & 4.6 \\
\hline Eaton-Littler ligament reconstruction & 17 & 3.9 \\
\hline Wilson's osteotomy & 14 & 3.2 \\
\hline Suspensionplasty with tendon, without trapeziectomy & 8 & 1.8 \\
\hline $\begin{array}{l}\text { Suspensionplasty with foreign material (e.g., Mini TightRope }{ }^{\circledR} \text { or } \\
\text { other suture), without trapeziectomy }\end{array}$ & 6 & 1.4 \\
\hline Arthrodesis of MC I and MC II & 0 & 0 \\
\hline Other & 15 & 3.4 \\
\hline
\end{tabular}

The sum of responses exceeds the total number of participants $(N=439)$, because more than one surgical procedure could be opted as a treatment of choice. In the second column, the responses are expressed as percentage of the total number of participants; these percentages thus do not add up to 100
When focusing on years of experience as hand surgeon, trapeziectomy alone was more often chosen by hand surgeons with less than 5 years of experience, compared to hand surgeons with more than 5 years of experience ( $30 \%$ vs $18 \% ; P=0.026)$. Prosthetic joint replacement on contrary was opted more often by hand surgeons with more than 5 years of experience, compared to hand surgeons with less than 5 years of experience ( $28 \%$ vs $11 \%, P=0.002)$. Among other surgical procedures, no relation was found with years of experience as hand surgeon.

\section{Discussion}

This European-wide survey shows that at present, trapeziectomy with LRTI is the most popular primary surgical treatment for isolated CMC-1 OA among participating hand surgeons in Europe, chosen by $46 \%$ as a preferred surgical treatment. In the USA, trapeziectomy with LRTI is also the most popular procedure, with percentages of surgeons choosing this technique as their favorite varying between $55.7 \%$ and $67.6 \%$ [4-6]. However, it seems that in the past decade, this procedure has become less popular with percentages dropping from $67.6 \%$ in a survey of 2010 , to $55.7 \%$ in a survey of 2017 .
In the meanwhile, trapeziectomy with suspensionplasty, either with suture (12.4\%) or with APL (15.4\%), gained popularity [5]. Trapeziectomy without any complementary procedures was chosen by $21 \%$ of the respondents in our survey as being part of their preferred surgical treatment. In the USA, this procedure was chosen by only $8 \%$ of the respondents as their preferred surgical procedure [4-6]. Even though studies have shown that trapeziectomy only is associated with fewer complications compared to trapeziectomy with additional LRTI [2, 3 ], the most recent US survey reported that only $15 \%$ of the respondents changed to trapeziectomy alone, from $24 \%$ of all surgeons that have changed procedures in the last 5 years [5].

In our study, one of the most remarkable findings was that $97 \%$ and $72 \%$ of the participating hand surgeons in France and Belgium mentioned that prosthetic joint replacement was one of their procedures of first choice. This is more than in all other European countries $(P<0.0001)$ and the USA, where only 0.9 $1,7 \%$ of the surgeons chose this procedure as their preferred technique. Furthermore, trapeziectomy with tendon interposition is less popular among US surgeons compared to European surgeons. In a US survey in 2012, 16\% of the respondents chose this procedure, while this was $5.5 \%$ in the survey held in 2010. In the last survey (2017), this item was not asked 
for. The evident differences between surgeons practicing in different countries are remarkable. It would be interesting to understand the varieties between the choices of surgeons in different countries. One study suggested that surgeons outside the USA rely more on current evidence, compared to surgeons in the USA [5]. A given reason was that less US hand surgeons work in an academic hospital setting, while this percentage would be higher in many international countries as a result of universal health care. In Europe, health care is universal and predominantly paid through taxes (Beveridge model), employer and employee payroll deductions (Bismarck model), or a combination of both models [7]. In our study, exact calculations comparing health systems to the surgical procedure are not made, as among the participating European countries, the Bismarck model is by far the most prevalent, resulting in unevenly distributed groups for comparison. Whether health care systems indeed are related to the choice of surgical procedure may be an interesting question for future studies. Besides the geographical trends, the choice of preferred procedure appears to be related to the number of years of experience. Surgeons with more than 5 years of experience significantly perform trapeziectomy alone less often compared to surgeons with less than 5 years of experience. The latter group possibly being more aware of evidence-based practice or yet being less experienced with the more complex procedures. Prosthetic joint replacement appears to be more popular among surgeons with more than 5 years of experience, which partly may be related to the difficulty of the procedure.

A limitation of our study is that not all member states of the FESSH participated, as approval for distributing the survey was not given by all countries. Moreover, it is not clear how many hand surgeons received the survey eventually, as for most participating member states, the exact number of members were unclear and the databases mostly also included hand therapists, residents and other members than hand surgeons. In the USA, an administrative database study on the national trends of surgical treatment of CMC-1 OA was performed [8]. In this study, a multinomial logistic regression model was used to assess the association between patients' characteristics and surgical treatment on a random 5\% sample of Medicare fee-for-service beneficiaries diagnosed with CMC-1 OA between 2001 and 2010. Also, surgeons' unique identifiers were used to examine their change of practice preferences over time. A similar study in Europe may help us obtaining more insight in the surgical practice among hand surgeons in Europe, including factors influencing the decision-making among the surgeons.

Our study explored the prevailing primary surgical procedures for isolated CMC-1 OA among hand surgeons in Europe. Geographical trends seem to play a large role in the choice of preferred technique. Compared to the USA, more alternative procedures are performed by the participating hand surgeons in Europe, including prosthetic joint replacement, trapeziectomy alone and trapeziectomy with other complementary procedures than LRTI. Similar to the USA, trapeziectomy with LRTI remains the most performed procedure among the participating hand surgeons in Europe. Factors influencing the decision-making process should be explored more closely in future studies, possibly using a European-wide administrative database study.

Funding This study received no funding.

\section{Compliance with ethical standards}

Conflict of interest Qi Yin, Merel J.-L. Berkhout, and Marco J. P. F. Ritt declare that they have no conflict of interest.

Ethical approval All procedures performed in studies involving human participants were in accordance with the 1964 Helsinki declaration and its later amendments or comparable ethical standards.

Informed consent Informed consent was obtained from all individual participants included in the study.

Open Access This article is distributed under the terms of the Creative Commons Attribution 4.0 International License (http:// creativecommons.org/licenses/by/4.0/), which permits unrestricted use, distribution, and reproduction in any medium, provided you give appropriate credit to the original author(s) and the source, provide a link to the Creative Commons license, and indicate if changes were made.

\section{References}

1. Haara MM, Heliövaara M, Kröger H et al (2004) Osteoarthritis in the carpometacarpal joint of the thumb. Prevalence and associations with disability and mortality. J Bone Joint Surg Am 86:1452-1457

2. Vermeulen GM, Slijper H, Feitz R, Hovius SE, Moojen TM, Selles RW (2017) Surgical management of primary thumb carpometacarpal osteoarthritis: a systematic review. J Hand Surg [Am] 36:157-169

3. Wajon A, Vinycomb T, Carr E, Edmunds I, Ada L (2017) Surgery for thumb (trapeziometacarpal joint) osteoarthritis. Cochrane Database Syst Rev 4:CD004631

4. Brunton LM, Wilgis EF (2010) A survey to determine current practice patterns in the surgical treatment of advanced thumb carpometacarpal osteoarthrosis. Hand (NY) 5:415-422

5. Deutsch Z, Niedemeier SR, Awan HM (2017) Surgeon preference, influence and treatment of thumb carpometacarpal arthritis. Hand (NY) 13:403-411

6. Wolf JM, Delaronde S (2012) Current trends in nonoperative and operative treatment of trapeziometacarpal osteoarthritis: a survey of US hand surgeons. J Hand Surg [Am] 37:77-82

7. Gaeta M, Campanella F, Capasso L et al (2017) An overview of different health indicators used in the European health systems. J Prev Med Hyg 52:114-120

8. Yuan F, Aliu O, Chung KC, Mahmoudi E (2017) Evidence-based practice in the surgical treatment of thumb carpometacarpal joint arthritis. J Hand Surg [Am] 42:104-112

Publisher's note Springer Nature remains neutral with regard to jurisdictional claims in published maps and institutional affiliations. 